\title{
Dark matter annihilation and decay factors in the Milky Way's dwarf spheroidal galaxies
}

\author{
Vincent Bonnivard* \\ LPSC, Université Grenoble-Alpes, CNRS/IN2P3, 53 avenue des Martyrs, 38026 Grenoble, \\ France \\ E-mail: bonnivardelpsc.in2p3.fr
}

\section{Céline Combet}

LPSC, Université Grenoble-Alpes, CNRS/IN2P3, 53 avenue des Martyrs, 38026 Grenoble, France

E-mail: combet@lpsc.in2p3.fr

\section{David Maurin}

LPSC, Université Grenoble-Alpes, CNRS/IN2P3, 53 avenue des Martyrs, 38026 Grenoble,

France

E-mail: dmaurin@lpsc.in2p3.fr

\section{Matthew G. Walker}

Department of Physics, Carnegie Mellon University, Pittsburgh, PA 15213, USA

McWilliams Center for Cosmology, 5000 Forbes Avenue Pittsburgh, PA 15213, USA

E-mail: mgwalkereandrew.cmu.edu

The Milky Way's dwarf spheroidal (dSph) galaxies are among the best targets for the indirect detection of dark matter (DM) from annihilation (resp. decay) products. The expected gammaray flux depends on the so-called ' $J$-factor' (resp. ' $D$-factor'), the integral of the squared DM density (resp. DM density) along the line-of-sight. Using a large number of simulated dSphs, we define an optimized Jeans analysis setup for the reconstruction of the DM density with stellarkinematic data. Employing this setup, we provide estimates of astrophysical $J$ - and $D$-factors for twenty-two Galactic dSphs, including the newly discovered Reticulum II. We finally identify several criteria that may indicate a contamination of a kinematic dataset by interlopers, leading to unreliable astrophysical factors. We find that the kinematic sample of Segue I, one of the closest $\mathrm{dSph}$, might be affected by this issue.

The 34th International Cosmic Ray Conference,

30 July- 6 August, 2015

The Hague, The Netherlands

\footnotetext{
* Speaker.
} 


\section{Introduction}

Owing to their large dynamical mass-to-light ratios, proximity, and low astrophysical backgrounds [1, 2, 3], the Milky Way's dwarf spheroidal (dSph) satellites are among the best targets for 'indirect' searches for particle dark matter (DM), via observations of gamma-rays that may be produced in annihilation or decay events (e.g., $[4,5,6])$. The constraints obtained with these objects on the thermally-averaged self-annihilation cross-section of DM are among the most stringent to date, e.g. with $\langle\sigma v\rangle<3 \times 10^{-26} \mathrm{~cm}^{3} \mathrm{~s}^{-1}$ (95\% CL) obtained by the Fermi-LAT collaboration [7] for DM particles with a mass below $100 \mathrm{GeV}$ annihilating into the $b \bar{b}$ channel.

From a given photon flux (or non-detection), one must estimate the density of DM in the source in order to set these contraints. This motivates observations and dynamical analyses of the tiny stellar populations that, for most of these objects, represent the only viable tracers of gravitational potentials. In many studies, specific forms of DM density profiles are fitted directly to the kinematic data of the dSph under scrutiny [8, 9, 10, 11], while others use 'cosmological priors' from structure formation simulations [12, 13]. Here, we focus on data-driven analyses that rely on parametric solutions to the spherical Jeans equation (e.g., $[15,14,16])$.

Using a large number of simulated dSphs, we define an optimized setup that allows us to mitigate possible biases of the analysis, and we investigate the impact of foreground contamination of the stellar-kinematic samples on the reconstruction of the $J$-factors. We then reconstruct the astrophysical factors of twenty-two Galactic dSphs, including the recently discovered Reticulum II (Ret II), for which a possible gamma-ray signal has been observed $[17,18]^{1}$. We finally find that Segue I (Seg I), often considered among the best targets, might suffer from contamination, leading to a possibly unreliable $J$-factor.

\section{Jeans analysis and astrophysical factors}

\section{$2.1 J$ - and $D$-factors}

The differential $\gamma$-ray flux coming from DM annihilation (resp. decay) in a dSph galaxy is proportional to the so-called 'astrophysical factor' $J$ (resp. $D$ ) [20],

$$
J=\iint \rho_{\mathrm{DM}}^{2}(l, \Omega) d l d \Omega \quad\left(\operatorname{resp} . D=\iint \rho_{\mathrm{DM}}(l, \Omega) d l d \Omega\right),
$$

which corresponds to the integration of the DM density squared (resp. DM density) along the line-of-sight (1.o.s.) and over the solid angle $\Delta \Omega=2 \pi \times\left[1-\cos \left(\alpha_{\text {int }}\right)\right]$, with $\alpha_{\text {int }}$ the integration angle. All calculations of astrophysical factors are done with the CLUMPY code [21], which has been upgraded with a Jeans analysis module in its second release [22].

\subsection{Jeans analysis: an optimized setup}

\subsubsection{Jeans analysis}

Assuming steady-state, spherical symmetry, and negligible rotational support, second-order

\footnotetext{
${ }^{1}$ Using proprietary data, the Fermi-LAT collaboration [19] published simultaneously an analysis of eight recently discovered dSphs, including Ret II, and did not find any significant excess.
} 
Jeans equation from the collisionless Boltzmann equation reads [23]:

$$
\frac{1}{v} \frac{d}{d r}\left(v \overline{v_{r}^{2}}\right)+2 \frac{\beta_{\mathrm{ani}}(r) \overline{v_{r}^{2}}}{r}=-\frac{G M(r)}{r^{2}}
$$

with $v(r)$ the stellar number density, $\overline{v_{r}^{2}}(r)$ the stellar radial velocity dispersion, $\beta_{\text {ani }}(r) \equiv 1-\overline{v_{\theta}^{2}} / \overline{v_{r}^{2}}$ the velocity anisotropy, and $M(r)$ the mass, dominated by DM, enclosed within radius $r$. After solving Eq. (2.2) and projecting along the 1.o.s., the (squared) velocity dispersion at the projected radius $R$ reads

$$
\sigma_{p}^{2}(R)=\frac{2}{\Sigma(R)} \int_{R}^{\infty}\left(1-\beta_{\text {ani }}(r) \frac{R^{2}}{r^{2}}\right) \frac{v(r) \overline{v_{r}^{2}}(r) r}{\sqrt{r^{2}-R^{2}}} \mathrm{~d} r,
$$

with $\Sigma(R)$ the surface brightness profile. We compare the l.o.s velocities of the stars to the projected velocity dispersion $\sigma_{p}$, using parametric forms for the unknown velocity anisotropy $\beta_{\text {ani }}(r)$ and DM density profile $\rho_{\mathrm{DM}}(r)$. We use the following likelihood function [12]

$$
\mathscr{L}=\prod_{i=1}^{N_{\text {stars }}}\left(\frac{(2 \pi)^{-1 / 2}}{\sqrt{\sigma_{p}^{2}\left(R_{i}\right)+\Delta_{v_{i}}^{2}}} \exp \left[-\frac{1}{2}\left(\frac{\left(v_{\mathrm{i}}-\bar{v}\right)^{2}}{\sigma_{p}^{2}\left(R_{i}\right)+\Delta_{v_{i}}^{2}}\right)\right]\right)^{P_{i}}
$$

which assumes a Gaussian distribution of 1.o.s. stellar velocities $v_{i}$, centered on the mean stellar velocity $\bar{v}$, with a dispersion of velocities (at the radius $R_{i}$ ) coming both from the intrinsic dispersion $\sigma_{p}\left(R_{i}\right)$ and the measurement uncertainty $\Delta_{v_{i}}$. The membership probabilities $P$ quantify, for each star, the probability of belonging to the $\mathrm{dSph}$. They can be used as weights, as in Eq. (2.4), or only to select the high probability members (e.g., with $P>0.95$ ). Finally, we obtain probability density functions (PDFs) of the anisotropy and DM parameters with a Markov Chain Monte Carlo (MCMC) engine, using the GreAT toolkit [24, 25], and then compute the median and credible intervals (CIs) of the astrophysical factors for a large range of integration angles.

\subsubsection{An optimized setup}

Many choices of parametrizations can be used for the DM density, velocity anisotropy and stellar number density profiles. In order to examine the impact of the various assumptions on the reconstruction of the astrophysical factors, we analyse three suites of mock data sets that consist of stellar positions and velocities drawn from static distribution functions that satisfy the collisionless Boltzmann equation. The first two suites are similar to the ones used previously by [26], [14], and [27]. They were obtained by varying different combinations of parametrizations of the Jeans ingredients (e.g. 'cusp' vs. 'core' DM densities, etc...). The third suite is available and described in detail as part of The Gaia Challenge ${ }^{2}$, and contains triaxial models that violate the common assumption of spherical symmetry.

We apply various Jeans analyses to each mock $\mathrm{dSph}$, and compare the reconstructed $J$ - and $D$ factors to their true values. From a thorough testing of the different ingredients, we have defined in [31] an optimized Jeans analysis setup which allows us to mitigate several possible biases. Biases coming from the light and anisotropy profiles are caused by too-constrained parametrizations, and can be addressed by using profiles with large degrees of freedom. We also find that using an Einasto

\footnotetext{
${ }^{2}$ http://astrowiki.ph.surrey.ac.uk/dokuwiki
} 
(e.g., [28]) or a Zhao-Hernquist [29, 30] parametrization for the DM profile gives similar results, given the small spatial range covered by stellar measurements. We give optimized ranges for the parameters priors of the Einasto profile, which has less free parameters than Zhao-Hernquist and allows for faster MCMC analyses. Finally, applying a spherical Jeans analysis to possibly triaxial dSphs adds a systematic uncertainty on the $J$-factor, which we estimate to be of order 0.4 dex. See $[31,32]$ for a precise description of the optimized setup.

\subsection{Contamination of stellar-kinematics}

The various tests mentioned above were undertaken on contamination-free mock dSphs, i.e. for which all the stars in the samples were dSph members. However, real stellar-kinematic datasets contain also foreground contamination from the Milky Way, which has to be separated from the bona fide members of the $\mathrm{dSph}$. The conventional approach to handle these interlopers relies on the arbitrary definition of some threshold separating members from outliers (sigma-clipping method, see e.g. [33]). Here, we focus on the expectation-maximisation (EM) algorithm [34], which gives more precise information in the form of membership probabilities $P$ for each star of the sample, and we test the reliability of $J$-factors estimates for various contamination levels.

We generate thousands of mock data sets that each sample mixtures of three simulated stellar populations tracing a gravitational potential dominated by DM. The first population represents bona fide members of a dSph galaxy. The second represents contamination from a tidal stream in which the dSph may be embedded, as may be the case if the object formed as the satellite of a more massive dSph that was more easily disrupted by Galactic tides [35]. The third represents contaminant stars in the Galactic foreground (see [36] for a precise description of the mock datasets). We then run a Jeans analysis on these mock dSphs, either using all the stars and weighting their contribution to the likelihood by their $P$ values (Eq. 2.4), or selecting only the stars with $P>0.95$. We find that:

- contaminated dSphs tend to show a large fraction of stars with ambiguous membership status $(0.05<P<0.95$, left panel of Fig. 1);

- stellar contamination can cause a large $J$-factor overestimation, up to several orders of magnitudes (right panel of Fig. 1);

- $J$-factors can be very different from a $P$-weighted to a cut-95\% analysis in presence of strong contamination levels.

These criteria can be used to detect a possible impact of contamination for real dSphs. This seems to be the case for Seg I, as described hereafter.

\section{Application to the Galactic dSphs}

\subsection{Contamination in Segue I?}

Seg I is often considered as one of the best target for indirect detection of DM [40], because of its proximity $(d \sim 23 \mathrm{kpc})$ and large inferred DM density. This object has been deeply observed 

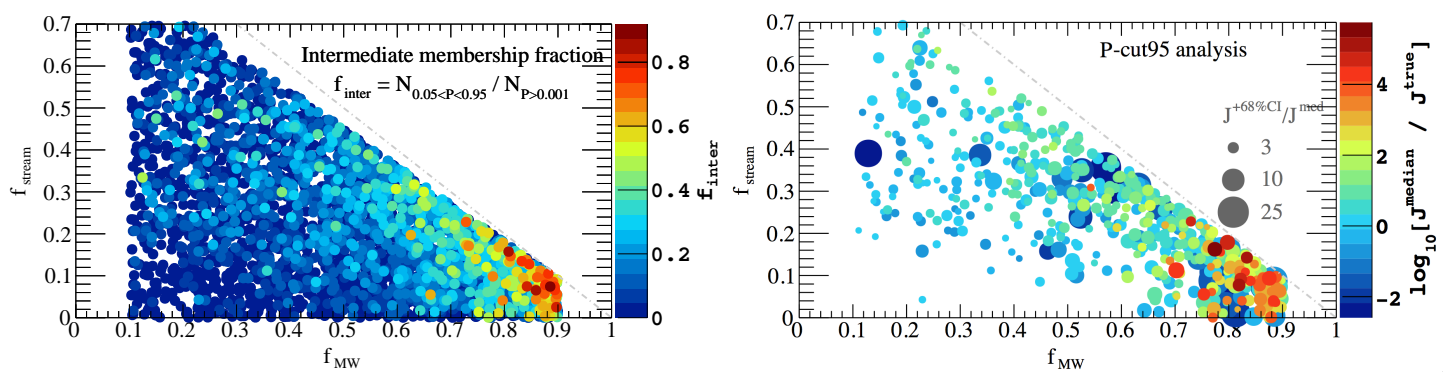

Figure 1: Left: fraction $f_{\text {inter }}$ of plausible members (membership probability $P>10^{-3}$ ) whose membership status is ambiguous $(0.05<P<0.95)$ for our 4000 mock data sets, as a function of the fractions of the sample contributed by contamination from the Milky Way ( $x$ axis) and stream ( $y$ axis). Right: accuracy of estimated $J$-factors (MCMC/cut-95\% analysis), for the 545 mock dSphs with $f_{\text {inter }}>0.1$. The colour scale shows deviation of the estimated $J$-factor from the true value. The sizes of the symbols correspond to the size of the $68 \% \mathrm{CI}$ on the estimated $J$-factor.
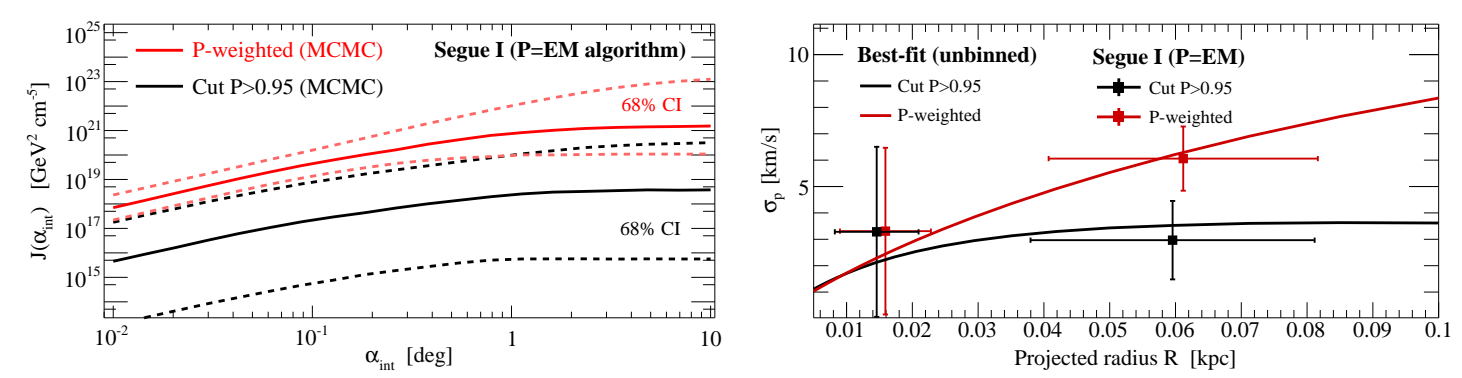

Figure 2: Left: $J$-factor of Seg I as a function of the integration angle, reconstructed either using all stars and weighting their contribution by their membership probability $P$ (red), or selecting only the stars with $P>0.95$ (black). Right: Same, but for the binned velocity dispersion profile. The best-fits are shown only for illustration purpose, as the analysis was done on unbinned velocities.

by Cherenkov telescopes such as VERITAS and MAGIC [39, 38], and gives the strongest limits to date from dSph galaxies for heavy DM candidates $\left(m_{\chi} \sim 500 \mathrm{GeV}\right)$.

However, we find that the available stellar-kinematic sample of Seg I (393 stars, see [40]) shows strong signs of contamination. About $20 \%$ of the plausible members $\left(P>10^{-3}\right)$ show an ambiguous membership probability $(0.05<P<0.95)$, and we find that the reconstructed $J$-factor can vary by up to two orders of magnitude from a $P$-weighted to a cut- $95 \%$ analysis (left panel of Fig. 2). This is due to a large increase of the velocity dispersion when including these ambiguous members (right panel of Fig. 2). This behaviour is similar to what we observed on contaminated mock data, and therefore estimates of Seg I's $J$-factors should be regarded with extreme caution. On the other hand, the same tests applied to Ret II and to the 8 most luminous ('classical') dSphs showed no sign of contamination, except for Fornax ${ }^{3}$. All the details of the analyses are presented in [36] and [32].

\footnotetext{
${ }^{3}$ Fornax shows both a large fraction of ambiguous members and a sensitivity to the treatment of the membership probabilities, but the effect is much less important than in Seg I.
} 


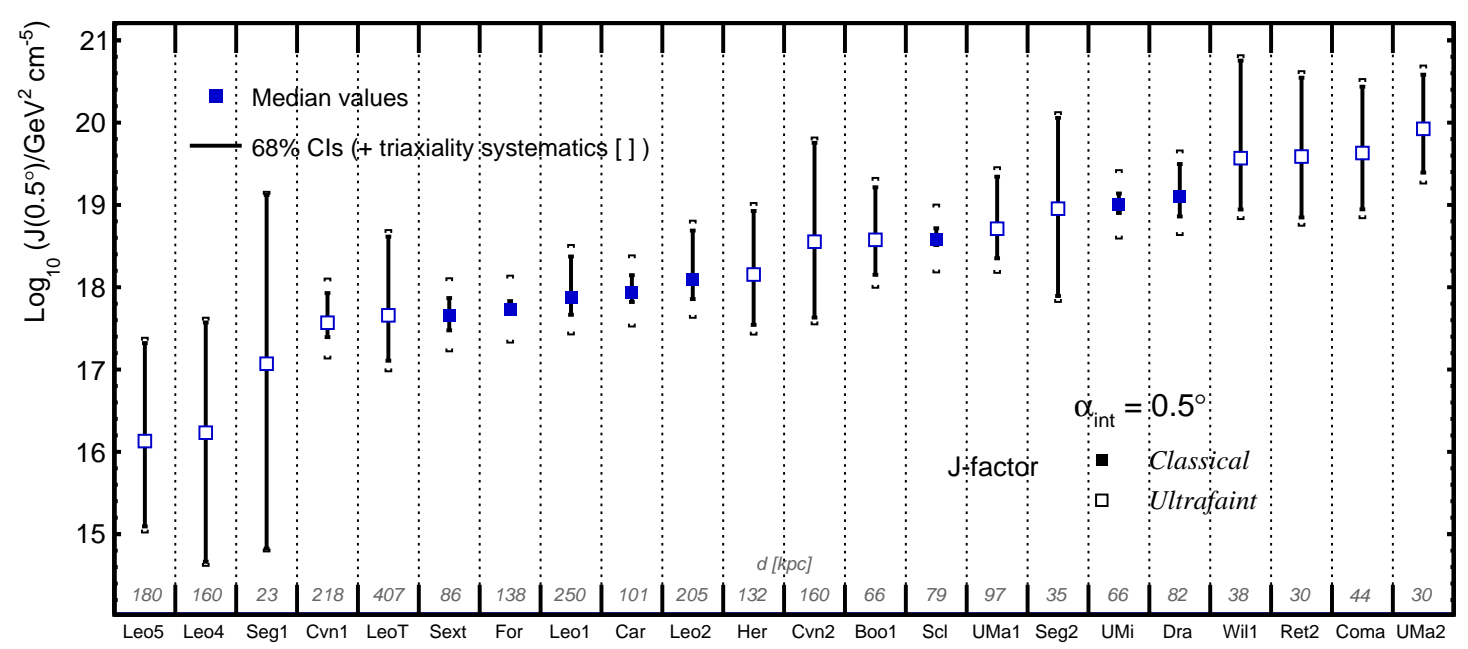

Figure 3: $J$-factors and $68 \%$ CIs for $\alpha_{\text {int }}=0.5^{\circ}$ : the '[]' symbols combine in quadrature the $68 \%$ statistical uncertainties and possible systematics $( \pm 0.4)$ from triaxiality of the dSph galaxies.

\section{2 $J$ - and $D$-factors for twenty-two dSphs}

We apply our optimized Jeans analysis setup to twenty-two Galactic dSphs, including eight 'classicals' and fourteen 'ultrafaints'. The latter objects were discovered more recently, and fewer stellar-kinematic measurements are available due to their very faint luminosities. Some of them are however very promising targets for indirect detection of DM because of their proximity. We provide estimates of both $J$ - and $D$-factors, as well as their uncertainties. The latter reflect realistically our degree of ignorance of these objects, because of the large flexibility allowed in our data-driven analysis for the DM density, velocity anisotropy and stellar number density profiles.

Fig. 3 shows the ranking of the targets, at the integration angle $\alpha_{\text {int }}=0.5^{\circ}$, for annihilating DM. The 'classical' dSphs UMi and Draco are confirmed as the potentially-brightest and most favoured targets in terms of $J$-factors. The 'ultrafaint' objects UMa 2, Coma, Wil 1 and Ret II outrank them, but suffer from larger uncertainties. Note that in the Southern hemisphere, Ret II ranks as the best target (see [37] for a detailed description of Ret II's analysis). For decaying DM, the ranking is very similar, with UMa 2 remaining the brightest 'ultrafaint' target at $\alpha_{\text {int }}=0.1^{\circ}$ (not shown, see [32]).

\section{Conclusion}

Dwarf spheroidal galaxies have been widely targetted in for searches for annihilating/decaying DM in the Galaxy. This has enabled gamma-ray telescopes to set stringent limits on the DM particle properties. Reliable estimates of their $J$ - and $D$-factors and associated error budgets are clearly crucial in this regard. This study extends and improves the reconstruction of the astrophysical factors for dSph galaxies, using an optimized Jeans analysis setup based on thorough testing on simulated dSphs. $J$ - and $D$-factors for eight 'classical' and fourteen 'ultrafaint' Galactic dSphs, including the recently discovered Ret II, are computed in a consistant way, and the objects are 
ranked according to their median estimates. We find UMi and Draco to be the most favoured targets in terms of magnitude and robustness of their $J$-factors.

Using contaminated mock dSphs, we also identify several characteristics of stellar-kinematic datasets suffering from foreground contamination, for which estimates of the $J$-factor can be unreliable. Such data sets tend 1) to have a large fraction of stars with ambiguous membership status and 2) to give very different $J$-factor estimates depending on how we treat these ambiguous stars. These characteristics are observed in the kinematic sample of the 'ultrafaint' dSph Seg I, for which astrophysical factor estimates should therefore be regarded with caution.

\section{Acknowledgements}

This work has been supported by the "Investissements d'avenir, Labex ENIGMASS" and by the French ANR, Project DMAstro-LHC, ANR-12-BS05-0006. MGW is supported by National Science Foundation grants AST-1313045, AST-1412999. This study used the CC-IN2P3 computation center of Lyon.

\section{References}

[1] Aaronson M., 1983, ApJ, 266, L11

[2] Mateo M. L., 1998, ARA\&A, 36, 435

[3] McConnachie A. W., 2012, AJ, 144, 4

[4] Lake G., 1990, Nature, 346, 39

[5] Evans N. W., Ferrer F., Sarkar S., 2004, Phys. Rev. D., 69, 123501

[6] Strigari L. E., 2013, Phys. Rep., 531, 1

[7] Fermi-LAT Collaboration, 2015, ArXiv e-prints, arXiv:1503.02641

[8] Bergström L., Hooper D., 2006, Phys. Rev. D., 73, 063510

[9] Sánchez-Conde M. A., Prada F., Łokas E. L., Gómez M. E., Wojtak R., Moles M., 2007, Phys. Rev. D., 76, 123509

[10] Bringmann T., Doro M., Fornasa M., 2009, Journal of Cosmology and Astro-Particle Physics, 1, 16

[11] Pieri L., Lattanzi M., Silk J., 2009, MNRAS, 399, 2033

[12] Strigari L. E., Koushiappas S. M., Bullock J. S., Kaplinghat M., 2007, Phys. Rev. D., 75, 083526

[13] Martinez G. D., Bullock J. S., Kaplinghat M., Strigari L. E., Trotta R., 2009, Journal of Cosmology and Astro-Particle Physics, 6, 14

[14] Charbonnier A., Combet C., Daniel M., Funk S., Hinton J. A., Maurin D., Power C., Read J. I., Sarkar S., Walker M. G., Wilkinson M. I., 2011, MNRAS, 418, 1526

[15] Walker M. G., Mateo M., Olszewski E. W., Peñarrubia J., Wyn Evans N., Gilmore G., 2010, ApJ, 710, 886

[16] Geringer-Sameth A., Koushiappas S. M., Walker M. G., 2015, ApJ, 801, 74

[17] Geringer-Sameth, A., Walker, M. G., Koushiappas, S. M., et al. 2015b, ArXiv e-prints, arXiv: 1503.02320 
[18] Hooper, D., \& Linden, T. 2015, ArXiv e-prints, arXiv:1503.06209

[19] Fermi-LAT Collaboration, The DES Collaboration, Drlica-Wagner, A., et al. 2015, ArXiv e-prints, arXiv: 1503.02632

[20] Bergström, L., Ullio, P., \& Buckley, J. H. 1998, Astroparticle Physics, 9, 137

[21] Charbonnier A., Combet C., Maurin D., 2012, Computer Physics Communications, 183, 656

[22] Bonnivard V., Hütten M., Nezri E., Charbonnier A., Combet C., Maurin D., 2015, ArXiv e-prints, arXiv: $1506.07628 \mathrm{~B}$

[23] Binney J., Tremaine S., 2008, Galactic Dynamics: Second Edition. Princeton University Press

[24] Putze, A. 2011, International Cosmic Ray Conference, 6, 260

[25] Putze, A., \& Derome, L. 2014, Phys.Dark Univ., doi:10.1016/j.dark.2014.07.002

[26] Walker M. G., Peñarrubia J., 2011, ApJ, 742, 20

[27] Walker M. G., Combet C., Hinton J. A., Maurin D., Wilkinson M. I., 2011, ApJ, 733, L46

[28] Merritt D., Graham A. W., Moore B., Diemand J., Terzić B., 2006, AJ 32, 2685

[29] Hernquist L., 1990, ApJ, 356, 359

[30] Zhao H., 1996, MNRAS, 278, 488

[31] Bonnivard V., Combet C., Maurin D., Walker M. G., 2015, MNRAS, 446, 3002

[32] Bonnivard V. et al., 2015, ArXiv e-prints, arXiv:1504.02048B

[33] Yahil A., Vidal N. V., 1977, ApJ, 214, 347

[34] Walker M. G., Mateo M., Olszewski E. W., Sen B., Woodroofe M., 2009, AJ, 137, 3109

[35] Belokurov V. et al., 2009, MNRAS, 397, 1748

[36] Bonnivard V. et al., 2015, ArXiv e-prints, arXiv:1506.08209B

[37] Bonnivard V. et al., 2015, ArXiv e-prints, arXiv:1504.03309B

[38] Aleksic J., Ansoldi S., Antonelli L., Antoranz P., Babic A., et al., 2014, JCAP, 2, 8

[39] Aliu E., et al., 2012, Phys. Rev. D., 85, 062001

[40] Simon J. D. et al., 2011, ApJ, 733, 46 